\title{
Gravitational Chemical Bond with Real Magnetic Charges and True Antielectrons
}

\author{
Robert A. Sizov \\ Moscow, Russia \\ Email: sizov.robert@gmail.com
}

How to cite this paper: Sizov, R.A. (2020) Gravitational Chemical Bond with Real Magnetic Charges and True Antielectrons. Open Journal of Physical Chemistry, 10, 48-67.

https://doi.org/10.4236/ojpc.2020.101003

Received: December 26, 2019

Accepted: February 10, 2020

Published: February 13, 2020

Copyright $\odot 2020$ by author(s) and Scientific Research Publishing Inc. This work is licensed under the Creative Commons Attribution International License (CC BY 4.0).

http://creativecommons.org/licenses/by/4.0/

\begin{abstract}
The detection by the author of real magnetic charges, as well as true antielectrons in of atomic structures allowed him to establish that atomic shells, as well as shells of nucleons are electromagnetic, and not electronic. Namely electromagnetic shells are the sources of gravitational field which is the vortex electromagnetic field. The elementary source of gravitational field is the electromagnetic quasiparticle (S-Graviton) which consists of two coupled dipoles (the magnetic and electric) rotating in antiphase in the same atomic or nucleonic orbit. Electrons in atomic shells are rigidly embedded in the compositions of S-Gravitons and, as a rule, cannot individually participate, for example, in processes of interatomic chemical bonding. Depending on the vector conditions the gravitational fields can be both paragravitational (PGF) so and ferrogravitational (FGF). The overwhelming number of atomic shells and all shells nucleons emit PGF. Between the masses (bodies, atoms, nucleons, etc.) emitting of PGF is realized a force of gravitational "Dark energy" pressing masses to each other. It is the compression of masses by forces of the gravitational "Dark energy" that lies at basis Physics of chemical bond. Depending on implementation in atoms of the effects intra-atomic gravitational shielding/lensing (IAGS/L) discovered and investigated by the author, the gravitational interatomic bonding mechanisms are divided into two groups: non-covalent bonds (IAGS effect) and covalent bonds (IAGL effect). Within the framework of the gravitational bond mechanism of the latter group which is implemented with participation paragravitational orbitals, such chemical concept as valence acquires a real physical meaning. The replacing the erroneous electronic concept of chemical bonding by the gravitational concept implies replacing the notion "electronegativity" of element by the notion the "gravitational activity" while maintaining existing quantitative ability of atoms in molecules to attract atoms of other elements.
\end{abstract}

\section{Keywords}

Magnetic Charges, Magnetons, Antimagnetons, True Antielectrons, Physical 
Mass, Vortex Electromagnetic (Gravitational) Field, "Dark Energy", Chemical Bonding, Valence

\section{Introduction to Physics of the Real Magnetic and Electric Charges, Gravitational Field and "Dark Energy"}

\subsection{The Real Magnetic and Electric Spinor Particles (Mono-Charges) Forming Atomic Shells}

The magnetic spinor particles (magnetic mono-charges) which are immediate sources of all magnetic fields and magnetic manifestations in Nature were discovered by the author in structures of atoms and substance and were first officially presented to scientific society in 2001 in publication [1]. It should be noted that the initial experiences which prompted the author to studies the problem participation of real magnetic charges in the structures of atoms and substance were his experiments with magnetic scattering of neutrons in ferrimagnetic crystals [2] [3]. The displacements of density so-called magnetic moments $\mathrm{Fe}^{3+}$ ions from nuclei found in these studies were qualified by the author as an indication on the possible existence of real magnetic charges in atomic shells.

In subsequent years the author performed a series of experiments that confirmed his preliminary conclusion and showed that the concepts of magnetic moments are the forced theoretical surrogates behind which hiding real magnetic charges are deftly hidden from us by Nature. In detail, experiments of the author in the problem, the real magnetic charges in atoms and substance are described in his publications [1] [4] and also briefly presented in the English-language article [5]. The results of the author's experimental and theoretical researches (1968-present) allowed him to assert that shells of atoms are not electronic, as was always assumed, but are electromagnetic. Below are brief descriptions of four real spinor particles (electric and magnetic charges) which, according to the author's research results, form the atomic shells.

Object No. 1 among the spinor particles that make up the shells of atoms is the electron, which in modern physical theories is forced to answer for both electrical and all magnetic manifestations. It is important to note that the detection and introduction of real magnetic charges, as well as of true antielectrons in the base physical representations release the electron from the need to be responsible for a physically alien it magnetic manifestations.

The magnetic spinors in compositions of the atomic shells (author's name for these magnetic particles is the magnetons) are fundamental particles of Matter which by their physical parameters are the magnetic analogs of electrons, i.e. having magnitude of charge and spin equal to corresponding values for electrons. Magnetons, like electrons, have the charge with negative sign $\left(\mathrm{g}^{-}\right)$, belonging to the class of leptons, and according to statistical properties to fermions (spin 1/2).

The magnetic antispinors (antimagnetons) which together with magnetons, electrons and true antielectrons enter into the compositions of the atomic shells 
are true antiparticles to magnetons. They have positive magnetic charge $\left(\mathrm{g}^{+}\right)$, i.e. are particles of Antimatter and belong to the class of antileptons. According to their statistical properties the antimagnetons are antifermions with spin $(-1 / 2)$ in relation to magnetons.

Note 1 . Here it is important to note that the first person who experimental observed real magnetic charges was the remarkable Felix Ehrenhaft who made his discoveries in the early 20th century [6]. The reasons which almost 150 years inhibit of the recognition of fundamental conclusions of Ehrenhaft discussed in detail in the publication of the author [7] and are noted in this article.

The true antielectrons, i.e. fundamental electric particles with charge $\mathrm{e}^{+}$which are neither positrons nor Dirac's "holes" [8] are the latter in the list of real spinor particles that form atomic shells. Story with the adventures of true antielectron in physical science is described in detail in the author's publication [9]. The true antielectrons which are particles of Antimatter should be classified as antileptons, and according to their statistical properties, as antifermions (spin is $-1 / 2$ relative to spin of electron).

The basic state of spinor particles is a state of the bispinor, i.e. of connected pair of spinor particles with charges opposite signs. Particles in this state are tightly pressed together what is accompanied by compensation of external activity their spins. Such of spinor pair can be called as the enclosed spinor dipole. The above ground state of spinor particles in the form of magnetic or electric bispinors does not lead to particle annihilation. The particle annihilation version was initiated by the results of the electron-positron interaction. However, the positron not is the true antielectron but is a varieties of Mass, i.e. it is of the atomic-like structure. The real antielectron "sits" in the positron structure as its nucleus and determines the electric charge of the positron. Upon contact with positron the electron combines with the true antielectron with forming of electric bispinor, and everything else from the composition of the positron in the form of the shallow electric and magnetic particles, most likely quarks, is ejected away. The atomic-shaped electromagnetic structure of positron is presented in the author's publication [10].

All the spinor particles mentioned above in the compositions of the electric and magnetic dipoles inhabit the orbits of atomic shells. Electromagnetic device shells of atoms that make up the Periodic Table of elements considered in the publication of the author's [11].

The main reason that real magnetic charges, more than a hundred years, are not recognized by a physical science is a physics of rigid confinement these particles in the structures of atoms and substance radically different from the confinement of electrons (see [4] [7]). It is the special conditions of confinement magnetic charges in atoms and substance that are the main reason for the formation of the vicious conception of the electric magnetism Maxwell [12] which is of result the superficial and exceptionally erroneous impression of Great Physicist from known experience Oersted.

However, the world scientific community adopted this erroneous impression 
as the ultimate truth and, following Maxwell, declared the moving electric charges to be direct sources of the magnetic field. At same time the true sources of the magnetic field, i.e. the real magnetic poles (magnetic charges) which are the real structural components of atoms and substance were "buried alive" [13].

Curious to notice here that even the famous Dirac monopole should be defined as an electrified theoretic construction, since its functioning as a source of magnetic field is impossible without the participation of electric current which is introduced into the monopole by means of Dirac filaments [14].

\subsection{The Electromagnetic Vortex Nature of Gravitational Field and Physical Essence of Mass}

On the basis of own investigation, the author has shown that electromagnetic shells of atoms are the natural sources (generators) of gravitation field which, in really, is the vortex electromagnetic field. The elementary source of the gravitational field is the vortex electromagnetic quasi-particle which received from the author the name S-Graviton ( $\mathrm{S}=$ source) (see [1] [5]).

The composition of the S-Graviton: two the spinors (electron and magneton) and two antispinor corresponding to them. Figuratively speaking the S-Graviton is a combination from of the electric and magnetic dipole rotating in antiphase on the same atomic orbit. This quasi-particle can also be represented in the form of two orbital currents electric and magnetic charges which in opposite phase move on one atomic orbit. Model representation of the vortex orbital EM-current, i.e. S-Graviton, must be written in the following form: $\operatorname{rot}\left[\boldsymbol{J}_{e}-\boldsymbol{J}_{g}\right]$ where $\boldsymbol{J}_{e}$ and $\boldsymbol{J}_{g}$ are vectors of instantaneous density of currents electric (e) and magnetic ( $\mathrm{g}$ ) charges in the composition of the orbital vortex electromagnetic current.

Then a equation process of gravitational field education through S-Graviton can be presented in the form: $k \operatorname{rot}\left[\boldsymbol{J}_{e}-\boldsymbol{J}_{g}\right]=\operatorname{rot}[\boldsymbol{E}-\boldsymbol{H}]$ (1), where $E$ and $H$ are vectors instantaneous strength of electric and magnetic fields in the composition of vortex electromagnetic (gravitational) field, and $k$ is the proportionality factor. The minus signs put in the above equation of the gravitational field education correspond both to antiphase of the orbital currents of electrical and magnetic charges, so and antiparallel orientation of vectors of instantaneous strength of electric and magnetic fields in every point of the gravitational field.

As follows from the above Equation (1), the gravitational field is the vortex electromagnetic field which is characterized by circular change of directions of vectors strength of electric and magnetic field ( $E$ and $H$ ) that are equal in magnitude and opposite in direction in relation to each other in each point. In contrast to the vortex magnetic field, to each point of which there corresponds one vector of instantaneous field strength $H$, every point of the elementary gravitational field is answered two vectors of instantaneous strength of fields $E$ and $H$ equal by value and oriented antiparallel each other.

Vortex magnetic field is product of the revolving spinor magnetic dipole. The elementary source of gravitational field is the S-Graviton which can be defined as dynamic EM-dibispinor, i.e. two coupled dipoles (electric and magnetic) ro- 
tating in antiphase on one atomic orbital.

Gravitational field которое испускает один или несколько S-Gravitons, отвечающее условию $\langle\operatorname{rot}[\boldsymbol{E}-\boldsymbol{H}]\rangle \neq 0$, by analogy with the ferromagnetism can be called ferrogravitational field (FGF). Gravitational field corresponding to the condition $\langle\operatorname{rot}[\boldsymbol{E}-\boldsymbol{H}]\rangle=0$, by analogy with the paramagnetism, can be called paragravitational field (PGF).

The real physics of the electromagnetic shell of atoms implies the mandatory inclusion of all the electrons existing in its compositions in structures of $\mathrm{S}$-Gravitons, the main property of which is the ability to produce a gravitational field. In this case a field of the electron is included in the composition of the vortex electromagnetic (gravitational) field as it's an electric component. Therefore, in theoretical operations with electrons in atomic shells, it is necessary to take into account the conditions for the hard binding of these particles in the compositions of S-Gravitons.

Since 2001 (see a publication [1]) the author argues that the Physical mass (PM), for example the atoms or nucleons, is the dynamic EM-complex consisting of electric and magnetic particles the main property of which is a generation of the gravitational fields. In other words, all that produces the gravitational field is mass. All spinor particles, that is, fundamental charged mono-particles, are massless, since they can't individually generate the vortex electromagnetic (gravitational) field.

\subsection{The World Physical Triad and "Dark Energy" in So-Called Interactions between Charges and Masses}

The results of years of researches real magnetic charges allowed the author to formulate the conception of the world Physical Triad (PT), according to which the Real World consists of three fundamental phases: Matter, Antimatter and Energo-phase (Energo-medium) [15] [16]. All spinor particles with negative charge belong to Matter, and particles with a positive charge constitute Antimatterial phase. Such names of spinor particles as "lepton" and "antilepton", as well as "fermion" and "antifermion" are determined by the natural essence of the particles, that is their material or antimaterial nature.

Note 2. It should be noted that in existing physical concepts, for example, atoms or nucleons are often referred to as matter. In his articles the author tries to explain that atoms, nucleons are not is matter at all. These structural formations consisting of material and antimaterial particles with electric and magnetic charges are, exclusively, varieties of physical mass. In addition, such physical essence as Antimatterial phase (Antimatter) under no circumstances can it be can't be antisubstance.

The Energo-phase (Energo-medium) is superhigh-density of gas-like medium that fills the entire World Space and consists of its own massless and spinless particles, which are called the Energions (author's title) and are designated as $\mathcal{E}$. It is important to note that the Energo-phase can also be referred to as Energo-ether. Can find the points of contact between the Energo-phase and the Physical vacuum. 
The energetic derivative from the Energo-phase is the so-called "Dark energy" ("DE") which is determined by the non-equilibrium states of Energo-phase in the form of local pressure fields $\left(P_{\varepsilon}\right)$ created by the Energions.

Figure 1 shows the scheme of the world Physical Triad which includes three fundamental world phases: Matter, Antimatter and Energo-medium (Energo-phase). The fundamental particles that constitute the Phases of the Triad are indicated on the diagram, i.e. the spinors, antispinors and energions. In Figure 1 marked and such derivatives from the phases of Triad, as the spinor fields and "Dark energy". Outside of this scheme remain are condensates consisting of spinor particles of Matter and Antimatter, called Masses.

The formations of "Dark energy" induce by spinor fields, i.e. fields of charged particles. Under this all varieties of spinor fields, including gravitational fields, themselves have no have essential of the force value. They are only intermediaries that influence on the local energy state of Energo-medium and induce the formation of "Dark energy" in it. Namely the "Dark energy" is global force factor which performs all the power acts with particles and masses, both in the scales of cosmos (the motion of galaxies, stars, planets, etc.), so and in the microcosm (the dynamics and so called interactions between Atoms and Charged Particles in compositions of Mass).

Between the Masses (atoms, bodies and other) which emit paragravitational field implemented the so-called the attraction or gravity. It is this last process, which is carried out, most often, by the forces of the gravitational "Dark energy" is the determining process in the physics of chemical bonding.

The Masses which emit the ferrogravtational field are repulsed from massessources of the paragravitational field, for example, from Earth, what constitute effect of the Gravitational levitation (GL) discovered and researched by author present article [17] [18]. An example of the so-called the ferrogravitational atom, i.e. atom emitting the FGF, is protium which is also called light hydrogen (designated as ${ }^{1} \mathrm{H}$ ). The manifestation of the protium volatility is determined precisely by the effect of gravitational levitation.

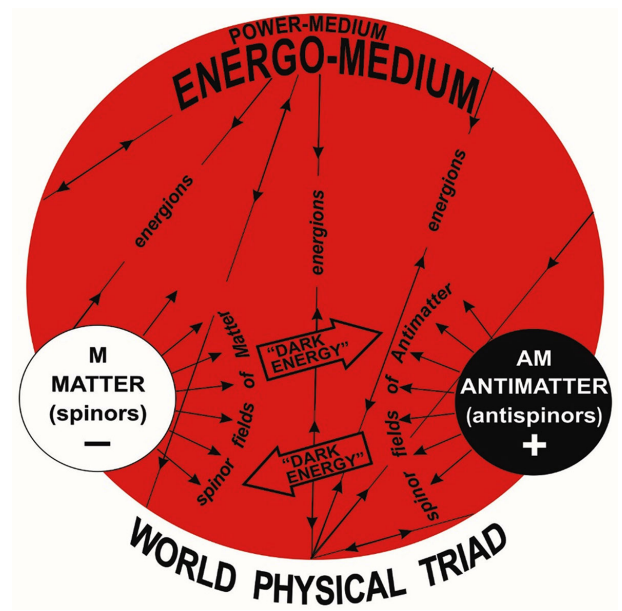

Figure 1. The diagram of the world physical triad. 
The measure of the gravitational mass is the magnitude of the force of the gravitational "Dark energy" ( $G$ "DE") which is realized, for example, in pairs of bodies or atoms. The equation of the so-called Law of universal gravitation should be written in the form $F_{\mathrm{DE}}= \pm G \frac{M_{1} \cdot M_{2}}{r^{2}}$, where the + sign refers to the case when the gravitational "DE" is implemented in of the pairs atoms or bodies emitting PGF. The masses, in this case, are pressed to each other by the force of the gravitational "DE" what is mistakenly perceived as their attraction. If the effect of gravitational levitation is realized between bodies or atoms what, as a rule, takes place in a pair of masses emitting PGF and FGF, then before the constant $\mathrm{G}$, in the above equation, a minus sign is set.

It is important to note that the forces of gravitational "DE" between masses over very small distances (e.g. less than 1 Angstrom) are not sufficiently studied. There is high probability that on small distances between the sources of the gravitational fields, the forces of the gravitational "Dark energy" are comparable with forces so called of electrostatic attraction and can even exceed its.

The reason that was closed all the possibilities of approaching of physical science to explaining real processes associated with the action of the gravitational "Dark energy" is distorted view about actual physics of the gravitational field. As is generally known, in the existent official physical approaches, the gravitational field, more than one hundred years, binds with curvature of the four-dimensional space-time. In addition, a limiting factor here was the lack in of the representations of the world Physical Triad which, according to the author, to a greater extent than the Physical vacuum or traditional Ether is meets the reality.

The purpose of this article is the transformation of the existing notions of the electronic chemical bonding in due to new physical circumstances which associated with of introduction in physical notions of real magnetic poles (magnetic charges), as well as true antielectrons. It is important to note that these last real particles, which make up 3/4 of all spinor particles in atoms and substance, stubbornly not recognized by modern physical science and are not used in the interpretations of physical effects and manifestations.

\section{Intra-Atomic Gravitational Shielding/Lensing and Paragravitational Orbitals in the Physics of Chemical Bond}

In article [19] the author presented physics of discovered by him effect of the intra-atomic gravitational shielding/lensing which includes two related effects: intra-atomic gravitational shielding (IAGS) and intra-atomic gravitational lensing (IAGL).

The effect of IAGS is that gravitational field of atomic nucleus formed by its nucleons is completely shielded by the shell of the atom and cannot go beyond it. The atomic shell in this case is the gravitational insulator for gravitational field of nucleus, which, is usually, is the paragravitational field. In the framework of 
the IAGS effect the external force manifestations by atoms determined exclusively by the "DE" forces which are induced of the spinor fields of the atomic shell. In addition to the gravitational field, the electromagnetic shells of atoms can emit such the spinor fields as a vortex magnetic field $(\operatorname{rot} H)$ and an electrostatic field of ions $(E)$.

The IAGL effect is realized when the gravitational field of the atomic nucleus overcomes through the gravitational resistance RG of the atomic shell and goes beyond the limits of the atom. In the process of going beyond the limits of an atom, the PGF of the nucleus undergoes lensing (forming) by the atomic shell and takes the form of the narrow gravitational "language" which given name the paragravitational orbital (PGO). This definition refers to the region in which is concentrated a density of the PGF of atomic nucleus beyond the atom. The name "orbital" is borrowed from electronic theories of chemical bonding since namely PGO, in reality, are replace such theoretical surrogates as electronic orbitals.

Note 3. In the framework of intra-atomic physics developed by the author, two different manifestations of gravitational lensing can be considered: gravitational lensing which determines full of screening of the gravitational field of the nucleus by the atomic shell (IAGL1) and process the formation of the gravitational field of the nucleus when it leaves the atom (IAGL2). The first effect in the author's publications is called IAGS, and we will stick to this name in the future. Regarding the effect of IAGL2, it will be referred to as IAGL.

The gravitational fields of the nearest masses have a significant influence on a process of exit of the nucleus field beyond the limits of the atomic shell in the processes of combining atoms into molecules. Such masses can be both neighboring atoms so and large bodies, in the form of cosmic bodies (planets, stars, and others), in the gravitational fields of which the considered effects of IAGS and IAGL are realized. In the most general terms, this influence consists in the effect of gravitational fields of the marked masses on the EM-shell of the atom, what leads to a decrease in the magnitude of its gravitational resistance $R_{G}$. In this case, the gravitational breakdown of the resistance $R_{G}$ of the atomic shell is carried out, as a rule, by means the PGO of nonmetals with the support of gravitational fields of other influential PGF sources.

The spatial distribution of gravitational fields induced by the protium atom (2a) and the deuterium atom ( $2 b$ ) is shown in Figure 2. If in protium atom realizes the persistent IAGS effect and the gravitational field of its nucleus-proton can't go beyond the atomic shell, then the IAGL effect is realized in the deuterium atom. The PGF strength of the deuterium nucleus formed by the proton and neutron is able to overcome the resistance $R_{G}$ of the atomic shell and go beyond the atom. Thus, if protium is involved in the chemical bonding processes exclusively through the gravitational field of its shell, then the deuterium atom, due to the IAGL effect, introduces additionally in this process the PGF of nucleus in the form of PGO. In the diagram shown in Figure 2 the Earth is represented as a source of PGF which conducive a processes of chemical binding with the participation of hydrogen atoms. 


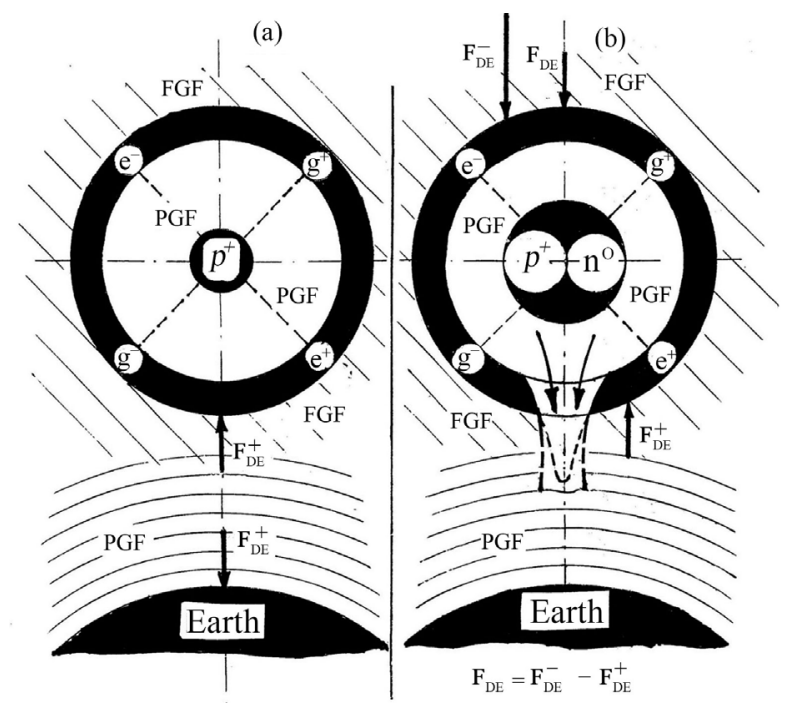

Figure 2. Schemes of distribution FGF and PGF under is conditions of realization of IAGS in the protium atom (2a) and IAGL in deuterium atom (2b). By black color in Figure 2, stand-alone sources of GF are indicated: electromagnetic atomic shells emitting FGF and their nuclei, i.e. proton (2a) and deyton (2b), emitting PGF. The concentric lines indicate the Earth's PGF. The forces of the "Dark energy" $\boldsymbol{F}_{\mathrm{DE}}^{-}$and $\boldsymbol{F}_{\mathrm{DE}}^{+}$, as well as their resultant a force $\boldsymbol{F}_{\mathrm{DE}}$ which manifests itself under the conditions of the IAGL effect in the deuterium atom are shown in Figure 2.

It should be recalled that the shells of hydrogen atoms are formed by one S-Graviton, which emits the ferrogravitational field. As noted above, FGF sources are repelled by PGF sources, such as Earth, which is the effect of Gravity levitation. It is the GL effect that is responsible for the manifestation of the volatility of atoms and molecules of ordinary hydrogen (protium). As for deuterium, the inclusion PGF of its nucleus in the chemical bonding process transfers this hydrogen to the so-called category of "heavy" elements. The arrows in Figure 2 show forces of positive "DE" $\left(\boldsymbol{F}_{\mathrm{DE}}^{+}\right)$and negative "DE" $\left(\boldsymbol{F}_{\mathrm{DE}}^{-}\right)$which are realized between the considered hydrogen atoms and the Earth. These forces carry out, respectively, the repulsion of the protium atom from the Earth (2a) and pressing of the deuterium atom to the Earth by the resultant force $\boldsymbol{F}_{\mathrm{DE}}(2 \mathbf{b})$. The existence of the latter force is due to the fact that the deuterium shell formed by one S-Graviton emits a ferrogravitational field. As a result the GL effect is realized and the deuterium atom is repelled from the Earth by the corresponding (positive) "DE" with force $\boldsymbol{F}_{\mathrm{DE}}^{+}$.

In order to simplify Figure 2(b) the diagram does not show the "DE" forces by which the deuterium atom presses the Earth to itself. These forces antisymmetric to forces are shown in Figure 2(b) and are equal to them in magnitude. The paragravitational field of the Earth affects the magnitude of the gravitational resistance of the $\mathrm{R}_{\mathrm{G}}$ of the atomic shell of deuterium and determines the possibility and direction of the output of the PGO of deuterium which, under the conditions of interaction of the gravitational fields of marked masses, is directed to the Earth (see Figure 2(b)). Important circumstance here is that PGO this 
paragravitational fields. The superposition of PGO of neighboring atoms leads to the formation of negative "DE" in the area of combining these fields of the forces of which press the atoms together. This process can be realized at considerable distances since the length of PGO can exceed atomic sizes. In addition, the density of PGF in PGO formulations can be very high.

The schemes of the processes of gravitational bonding of atoms in molecules that are realized with using PGO is shown below in Section 3, where gravitational covalent bonds are considered (see Figure 3). At the same time, one should not forget that in addition to PGO the atoms emit PGF which is induced them atomic shells. However, the "DE" forces induced by these last fields, under conditions of the implementation of gravitationally covalent bonds, reveal at short distances between the atoms and are most likely weak in comparison with the "DE" forces which are formed by PGO. Thus, PGO can be considered as a kind of gravitational "tentacles" which are engaged in the primary search and involvement in the composition of the molecules of the corresponding components from the totality of various atoms. As for the distance between the atoms from which the binding process of atoms begins under the action of the "DE" forces formed by PGO, we can assume that it can be several atomic diameters, i.e. be of the order of $\sim 2$ - 3 Angstrom.

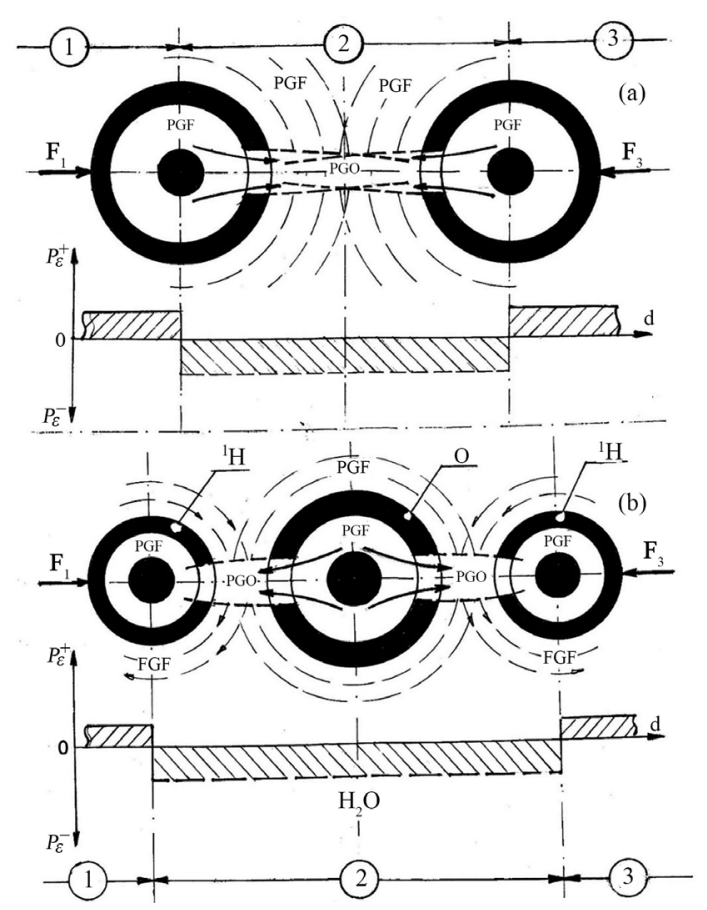

Figure 3. The schemes: of the covalent gravitational non-polar bond (3a) and gravitational donor-acceptor bond (3b). The black color in Figure 3 shows the atomic shells and nuclei of atoms whose gravitational fields involved in of processes of gravitational chemical bonding. Discontinuous concentric lines indicate the gravitational fields emitted by the shells of atoms. The resulting forces $F_{1}$ and $F_{3}$ are responsible for the interatomic chemical bond. The fields of atomic nuclei, which are source of PGFs, are shown in Figure 3 in white and are located both in intra-atomic regions and in PGO zones. In addition, in Figure 3 marked oblasts of FGF and PGF formed by atomic shells. 
Depending on the realization of the effects of IAGS and IAGL, atoms in the compositions of molecules, can be divided into two groups: gravitational donors and gravitational acceptors. The first group consists from atoms of non-metals which participate in interatomic bonding processes via external $\mathrm{PGO}_{\mathrm{s}}$. The example of gravitational donors is fluorine, oxygen, carbon and others. The participation the PGF of nuclei of acceptors in the formation of molecules is achieved by external breakdown of the resistance $R_{G}$ of atomic shells by means PGO of the nonmetal, i.e. gravity donors. Examples of the acceptors are metals and, in some cases, ordinary hydrogen, i.e. protium.

The existing concept of relative electronegativity (EN) of elements, in the concepts of physics of gravitational chemical bonds, is replaced by the concept of relative gravitoactivity of elements (GA). The gravitational activity is the property and quantitative characteristic of atom to carry out unifying gravitational influence with the atoms of other elements in the compositions of molecules. Herewith, the quantities $\chi$, the so-called of the electronegativity, attributed by the elements in existing system of electronic representations, without changing their values, transferred to the system of representations of gravitational chemical bonds. Such a transfer is quite correct, since the practical scale of electronegativity of atoms is based on the concept of covalent radius $r$, i.e. half the distance between the nuclei of atoms elements forming the covalent bond.

It is known that the electronegativity of an atom $(\chi)$ is a value proportional to the attractive energy of one valence electron located at a distance $r$ from the atomic nucleus, i.e. $\chi=(m+n) / r$, where $m$ and $n$ are the number of $\mathrm{p}$ and $\mathrm{s}$ electrons in the valence shell of atom. In principle, nothing prevents the use of covalent radii $r$ to determine the gravitoactivity of elements in the framework of the equation $\chi=(m+n) / r$, where $m$ and $n$ are the number of S-Gravitons in $\mathrm{p}$ and s-orbits in the outer so-called the valence shell of atom. It is easy to see that this gives the same result $\mathcal{X}$, since all the electrons in the atomic shells are part of S-Gravitons which, in its turn, are elementary sources of the gravitational field.

Thus, of the element fluorine $(F)$ corresponds highest GA among the elements of the periodic Table 1-6 periods equal to 4 , and element a cesium (Cs) corresponds to a minimum GA, equal to 0.7. In the system of gravitational chemical bonding of the element $F$ corresponds to the most significant in size and force activity PGO. As for cesium, it does not form external PGOs and the participation of PGF of its nucleus in chemical bonding processes occurs as a result of external breakdown of the gravitational resistance RG of its shell by means of PGO of gravitational donors, i.e. nonmetals.

\section{The Gravity-Covalent Bond and Valence of Chemical Elements}

The paragravitational orbitals ( $\mathrm{PGO}$ ) presented in the article are the natural substitutes for electronic orbitals used in existing electronic theories of chemical 
bonding, and the concept of atomic (gravitational) covalence is the natural substitute for the concept of atomic (electronic) covalence. In addition, the article noted that processes of chemical binding of atoms by means of PGO are the primary process which is implemented at considerable distances from the atom, due to the narrow focusing and high concentration of PGF in the composition of PGO. It should also be added that the "DE" forces induced by PGF of atomic shells also participate in atom bonding processes. However, the influence these forces are realized at shorter distances between atoms, in comparison with PGO.

Thus the process of interatomic chemical bonding is, at least, a double process. However, it should be borne in mind that the exit the PGF of nucleus beyond the atom during of gravitational lensing, as well as the processes of donor-acceptor gravitational coupling, can lead to significant changes in the magnitudes of the "DE" forces which are induced by the atomic shells. In other words, under conditions of heterogeneous chemical bonding, the "DE" forces induced by PGF of atomic shells can significantly differ from their values that are realized under conditions of mono-gravitational binding, for example, upon binding of metal atoms. It is also important to note that in addition to the gravitational "DE" forces noted above, the forces of electrostatic and magnetic "DE" can also participate in the processes chemical bonding of atoms.

In the most general sense, the gravitational covalent bond (GCB) is a bond of two or more atoms with the obligatory participation in the PGO process. Varieties of GCB are: non-polar, polar, as well as donor-acceptor gravitational bonds.

The nonpolar GCB are formed between atoms with the same physical parameters of the PGO emitted by them. In this case, the socialized density of PGO is symmetric with respect to the atomic nuclei $\left(\mathrm{O}_{2}, \mathrm{~N}_{2}, \mathrm{Cl}_{2}\right.$ and other). This bond is a real substitute for such a known type of chemical bond as electronic covalent non-polar bond. The polar CGB is formed between atoms which during chemical bonding processes emit PGOs differing in their physical parameters $\left(\mathrm{CO}_{2}\right.$, $\mathrm{CCl}_{4}$ and other).

When reorganizing the model of donor-acceptor electronic communication, as applied to the conditions of the action of gravitational mechanisms, it should be assumed that gravitational donors are atoms that are characterized by the formation of external PGO which realizing outside the atoms. Such donors are atoms of nonmetals. As for metals, so PGF of their nuclei cannot go beyond atoms and the participation these fields in covalent chemical bonding processes is carried out as a result of external breakdown of the gravitational resistance $R_{G}$ by means of PGO of the donor atoms. In this case, the PGO of the donor atom combines with the PGF of the nucleus in the volume of the metal atom, which plays the role of an acceptor in the system of gravitational chemical bond.

Thus, in the model presented, the PGO of atom of donor is transferred, for general use, to another atom which is a gravitational acceptor. The latter gravitational model, in some way, corresponds to the union of a pair of atoms by means of a common electron pair in a donor-acceptor model of electronic chemical 
bonding. This gravitational model of chemical bonding should be referred to as gravitational covalent donor-acceptor bonding.

Within the framework of described gravitational donor-acceptor model for PGO of nonmetals should be attributed a plus sign. As for gravitational acceptors, i.e. metal atoms due to the fact that their PGF does not go beyond atoms, their PGO should be, conditionally, determined with a minus sign. The possible gravitational acceptor may be atom of ordinary hydrogen (protium) in its compounds with nonmetals. The fact is that the persistent IAGS effect is realized in this atom and breakdown of gravitational resistance $R_{G}$ of its shell be realized through the action of neighboring the PGO of donor atoms in processes of chemical bonding.

Figure 3 shows the schemes: the covalent gravitational non-polar bond $\left(0_{2}, \mathrm{~N}_{2}\right.$, $\mathrm{Cl}_{2}$ and other) (3a) and gravitational donor-acceptor bond (3b). The scheme $3 \mathrm{~b}$ corresponds, for example, to the formation of a water molecule, i.e. $\mathrm{H}_{2} \mathrm{O}$. Each interatomic formation shown in Figure 3 has energy diagrams that determine the pressure levels $P_{\varepsilon}$ in the Energo-phase, as applied to regions 1, 2, and 3 in the gravitational chemical bonding processes under consideration. The designation of the PGO zones, as well as the $P_{\varepsilon}$ levels in Figure 3 by dashed lines is due to the fact that, at the moment, there are no exact data on both the PGO geometry and the ratio of $P_{\varepsilon}$ values and the shape of the corresponding lines on the energy diagrams.

The number of $\mathrm{PGO}_{\mathrm{s}}$ emitted by an atom in the formation of chemical compounds determines its valence.

Consequently, in relation to gravitational donors and acceptors, their PGOs can be both positive and negative, depending on whether they are external or internal relative to the atomic shell. For example, hydrogen in compounds with non-metals is capable of forming only one negative PGO, which corresponds to its valency of -1 . Such compounds are called covalent hydrides. Unlike hydrogen, a carbon atom can have four external, that is, positive PGO, which corresponds to its valency +4 .

\section{Gravitational "Dark Energy" in Physics of the Non-Covalent Chemical Bonding}

This chapter discusses the mechanisms of formation the chemical compounds from atoms, in which the full effect of IAGS is realized. This means that the chemical bond, in this case, is determined by the forces of "dark energy", which is induced by the spinor fields of only the atomic shells. In other words, in the mechanisms of interatomic bonds of this type of PGO and, therefore, a fields atomic nuclei do not participate. Such bonds are: metal, ionic and pure hydrogen bonds.

\subsection{The Metallic Bond}

The main factor of the force responsible for the formation of metallic com- 
pounds is the gravitational "Dark Energy" ( $G$ "DE"), which is formed under the influence of the para-gravitational fields of atomic shells of metal. Unlike the conditions of covalent bonding, such elements of chemical bonding as PGO are not involved in the physics of metallic bond and, therefore, the concept of valence here does not apply. Figure 4 shows the force diagram illustrating action of G "DE" in pair of so-called of the paragravitational atoms, in the present case, of atoms copper.

Along the vertical axis is represent pressure $P_{\varepsilon}$ in Energo-phase, and on horizontal axis a distance (d) between atoms. The oblast of low pressure in Energo-medium satisfying condition $P_{\varepsilon}<0$ in Figure 4 represents the oblast of negative "Dark energy". Forces $\boldsymbol{F}_{1}$ and $\boldsymbol{F}_{3}$ acting on atoms from external oblasts of the pair, where $P_{\varepsilon}>0$ carry out the compressing atoms to each other. The pressure that meets the condition $P_{\varepsilon} \geq 0$ corresponds to the average regional level $P_{\varepsilon}$ outside of oblast 2 marked in Figure 4.

It is the negative "Dark energy" that forms between the atoms of metals which emit of the paragravitational field is a universal force factor is responsible for the formation of metallic compounds.

The mechanism of gravitational chemical bonding, described in this section, is intended to replace such the model as the electronic metallic bond. First of all, there are no serious physical grounds for the assertion that free electrons in metals are a real connecting link between atoms. At the same time the free electron in metals is only factor for which the modern theory can lean when solving the problem of the metal bonding. Fields of free electrons (conduction electrons) in the metal lattice, in combination with the electric fields of ions, can generate both negative and positive "DE" forces of which, most likely, cancel each other out. In addition, one should not forget about the so-called mutual repulsion between negative ions in the metal lattice, which must be overcome by the forces of gravitational "DE". Thus, it is the gravitational "DE" is the main and constantly acting force factor determining the chemical bond in compounds of metal atoms.

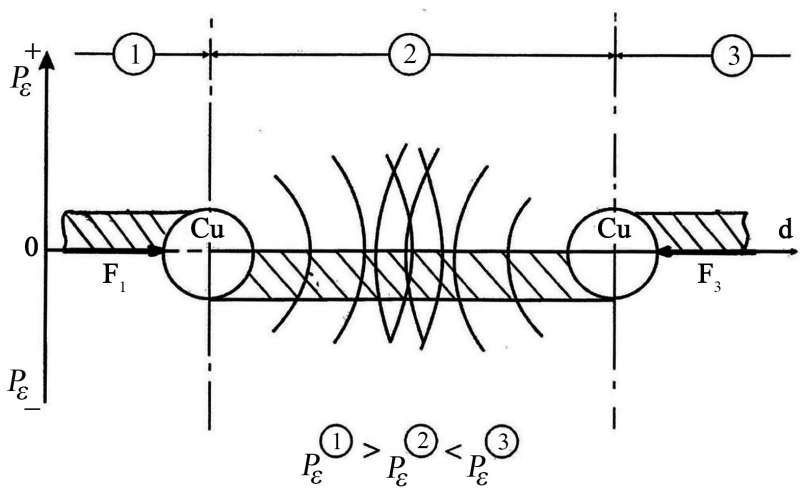

Figure 4. Gravitational "Dark energy" in the process of chemical bonding of the so-called paragravitational copper atoms. 
It should be added here that in addition to the gravitational "Dark energy" there is also the magnetic "Dark energy" induced by the vortex fields $\operatorname{rot} H$ of magnetic ions, as well as the electrostatic "Dark energy" can take part in processes of bonding in compounds of metals. When describing the gravitational metal bond, it should be taken into account that the forces of the gravitational "DE" formed by the fields of shells atomic are very significant and often exceed the bond strength which realizes in the conditions of gravitational covalent mechanisms. The latter circumstance is explained by the fact that the participation of the PGF of nucleus in chemical bonding in the form of PGO significantly weakens the gravitational "DE" forces induced by atomic shells.

\subsection{Electrostatic and Gravitational "Dark-Energy" in Ionic Bond Physics}

The only quasi-electronic process that is realized in the physics of chemical bonding is the ionic bonding. However, and in this process a forces that bind the ions are forces of electrostatic "Dark energy" (see, Appendix). In addition, the gravitational "Dark energy" necessarily participates in the processes chemical bonding and between ions since ions, as a rule, a source of PGF. The electrostatic "DE" which is formed between ions charged of the opposite electric charges is the second most significant force factor in chemical bonding systems after the gravitational "DE".

Figure 5 shows the force diagrams associated with the formation of negative "Dark energy" between electric ions which charged of charges of different signs (5a) and formation of positive "Dark energy" between charges of the same sign (5b). As noted above, these "Dark energy" must be named as an electrostatic "Dark energy", and it possible designation is E "DE". Vectors $\boldsymbol{S}_{\mathrm{sp}}$ and $\boldsymbol{S}_{\text {asp }}$ are the spin-vectors of ions, respectively, of spinors (sp) and antispinors (asp), which determine direction of propagation of their electrical fields. The forces $\boldsymbol{F}_{1}$ and $\boldsymbol{F}_{3}$ in Figure 5(a), as well as forces $F_{2}$ in Figure 5(b) are forces, respectively, of the negative and positive "Dark energy" which in given combination of electrostatic fields, pressing or repel the atoms in the pairs presented.

Since all kinds of physical mass are electromagnetic structures, i.e. consist of electric and magnetic charges, in addition to electrostatic "Dark energy" must exist and the magnetostatic "Dark energy" (Ms "DE") which is implemented between magnetic charges, as well as between the ions which charged by the magnetic charges. However, as shown in the publications of the author [8] [10], the formation of isolated magnetic charges (not associated in the pairs of + and - as well as the magnetic ionization of atoms is energetically very expensive process. Therefore, the magnetostatic "Dark energy", is likely, not enter in among the significant determinants of chemical bond, at least, in our electrical World [17].

It is important to note here that ions, as a rule, are sources of PGF and therefore in compounds with ions in addition to the electrostatic "Dark energy", necessarily binding forces due to the gravitational "Dark energy" are realized. It is forces of gravitational and electrostatic "Dark Energy" that determine the conditions of binding of atoms to take place in ionic crystals. 


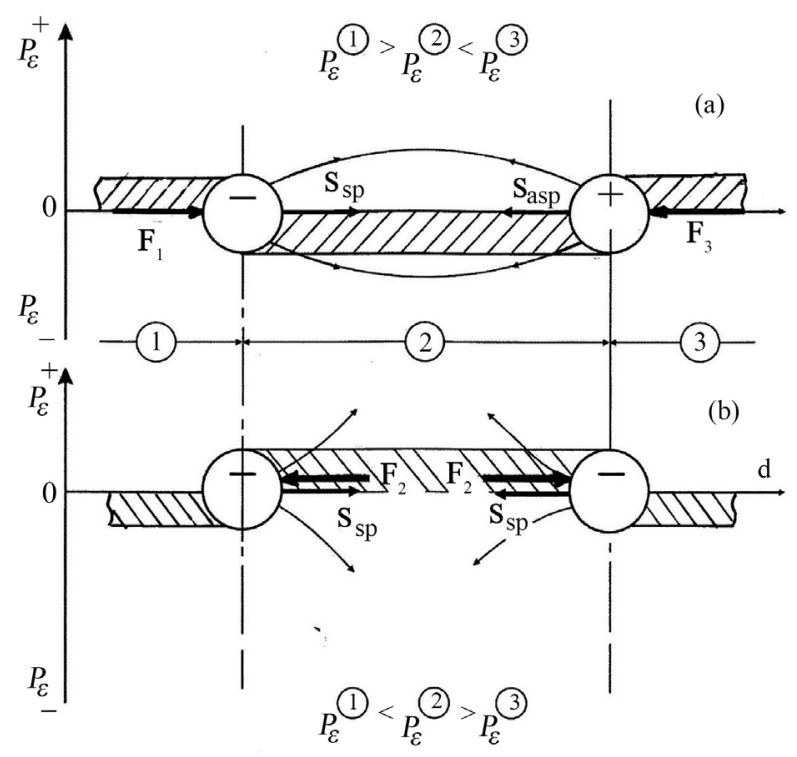

Figure 5. The models and the force effects of the negative and positive "Dark energy" in a pairs of ions with charges of different signs (5a) and of the charges of one sign (5b).

However, and the last set not is of full. In compounds with magnetic ions, for example, with iron ions is realized still and magnetic "Dark energy" ( $M$ "DE") which induced by magnetic fields of ions which should not be confused with the above mentioned of the magnetostatic "Dark energy".

\subsection{The "Dark Energy" in Processes of Bonding Atoms in Molecules $\mathrm{H}_{2}$ (Pure Hydrogen Bond)}

Above in this chapter two types of non-covalent chemical bonds that are realized under the influence of negative "Dark energy" inducing by paragravitational and electrostatic fields emitted by atomic shells were considered. However, there are opportunities for the formation of negative "Dark energy" and, therefore, chemical bonds between atoms whose shells emit a ferrogravitational field.

For example the formation of a hydrogen molecule $\left({ }^{1} \mathrm{H}_{2}\right)$ is determined by negative "Dark energy" which is realized between two atoms of protium the gravitational fields of which are ferrogravitational and differ only by in signs \pm of the vortex vectors $\operatorname{rot}[\boldsymbol{E}-\boldsymbol{H}]$. As shown in [19] IAGS effect is realized in the protium atom. Herewith the external powers manifestations by the atom protium are determined by "Dark energy" which induced by field its atomic shell, i.e. the S-Graviton. Recall that S-Graviton is the elementary source of the gravitational field which is of the ferrogravitational field. The chemical bond which realized between two protium atoms should be called the true hydrogen non-covalent gravitational bond. It is this variant of the hydrogen bond that is responsible for the formation of the ${ }^{1} \mathrm{H}_{2}$ molecule and, possibly, $\mathrm{He}_{2}$.

Figure 6 shows the force diagram corresponding to compression (condensation) of two hydrogen atoms in molecule ${ }^{1} \mathrm{H}_{2}$. The forces $F_{1}$ and $F_{3}$ in Figure 6 , which is responsible for the binding of atoms in molecule, are the product of the negative "Dark energy" is formed in the interatomic oblast. 


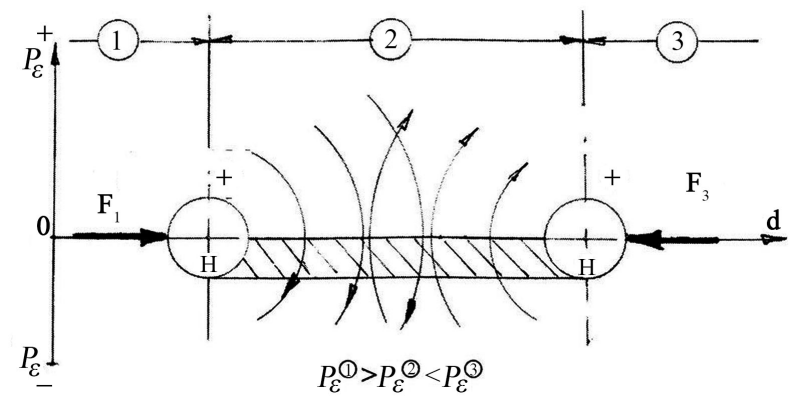

Figure 6. The energy diagrams corresponding compression of two hydrogen atoms in molecule ${ }^{1} \mathrm{H}_{2}$.

Unlike from gravitational compression of the "paragravitaitional" atoms, during the formation of molecules by the "ferrogravitational" atoms the most important factor is the mutual orientation of the vortex vectors its gravitational fields. The signs + in Figure 6 denote the orientation of the vortex vectors $\operatorname{rot}[\boldsymbol{E}-\boldsymbol{H}]$ of the gravitational fields, emitted by the atoms of hydrogen in the molecules ${ }^{1} \mathrm{H}_{2}$, those show that these vectors have the same direction. In this case, the strength vectors of ferrogravitational fields of neighboring atoms are in antiphase what is always accompanied by formation of negative "Dark energy" between them.

It should be noted that the molecules ${ }^{1} \mathrm{H}_{2}$ and $\mathrm{He}_{2}$ are unique examples of associations so-called of the ferrogravitational atoms under conditions of realization of complete shielding of the gravitational field their nuclei. The atoms of hydrogen and helium in these compounds do not exhibit properties that can be attributed to such concepts as valence or covalence.

In a huge number of chemical compounds with non-metals, hydrogen exhibits a property that corresponds to its valency equal -1 . This, the last property is associated with the violation of the conditions of complete IAGS in the process of chemical binding.

It is important to note that the purely hydrogen bond described in this section, takes place at bonding of the so-called ferrogravity atoms, the physics of which demonstrates only of the IAGS effect. Such atoms are protium and so called light helium (helium-4). In compounds with paragravity atoms, for example, with atoms of nonmetals, ferrogravity atoms behave like gravitational acceptors in donor-acceptor covalent bonds with a valency -1 .

\section{Conclusions}

The article presents the physical basics, as well as the procedure for replacing the existing electronic models of chemical bond on the really acting in nature of gravitational mechanisms. The need for such a replacement is explained by the fact that in physical science in result of various reasons the vicious concept of pure electron shell of atoms has been formed. It is this concept that underlies the formation of existing electronic theories of chemical bonding. However, the studies of F. Ehrenhaft and the present author showed that in addition to electrons 
in the shells of atoms, as structural components, exist are three more of fundamental mono-particles: two magnetic (magneton and antimagneton) and one electric particle, i.e. the true antielectron with charge $\mathrm{e}^{+}$. Paradoxical as it may seem but these three real fundamental particles are not acknowledged by existing physic science and are not included in the Standard Model.

The introduction of these unrecognized spinor particles into basic physics allows to find out the true nature of the gravitational field, as well as the role of electrons in its formation. As shown in Section 1.2 above, all electrons in atomic shells are rigidly connected in the compositions of the electromagnetic quasiparticles or S-Gravitons which are elementary sources of the vortex electromagnetic (gravitational) field. Thus, if You want to attract an electron from the atom's shell, including the valence shell, for any interactions, You should understand that the "tail" of three associated with it unrecognized particles, necessarily stretches behind it. In other words, whether You want it or not, but instead of an individual electron from the atomic shell You, necessarily, will have to deal with S-Graviton.

An important factor that allowed the author to approach the real physics of chemical bonding is the world system of fundamental particles discovered and studied by him, called as the World Physical Triad. It is the concept of the World physical Triad that allows us to find out the physical essence of spinor fields, as well as to determine their role in the formation of such a global force factor as "Dark energy". In addition, it would be impossible to explain the real physics of chemical bonding without including the effects of intra-atomic shielding and lensing in the physical representations. These effects, discovered by the author, belong to basic characteristics of atoms, without which getting closer to real atomic and interatomic physics is impossible.

\section{Acknowledgements}

The author expresses deep gratitude to Elena Sizova and Vitaly Sizov who for many years helped and supported him which largely contributed to the implementation of his research activities.

The author is grateful to the technical specialists: Alexander Davydov, Nikolai Koshelev, Alexander Lomakin, Evgeni Moiseenkov and Sergei Staritsyn who together with him conducted the main experiments connected with the discovery and investigation of real magnetic charges.

\section{Conflicts of Interest}

The author declares no conflicts of interest regarding the publication of this paper.

\section{References}

[1] Sizov, R.A. (2001) New Presentation of Nature Magnetism, Gravitation and Nuclear Forces of Bonding. Akademizdat Center "Science", Moscow, 62 p.

[2] Sizov, R.A. (1971) The Shift of the Magnetic Moment of the $\mathrm{Fe}^{3+}$-Ions from Nuclei 
in Z-Type Hexagonal Ferrite. Solid State Physics, 13, 2081-2088.

[3] Sizov, R.A. (1971) Delocalization of the Magnetic Moment of $\mathrm{Fe}^{3+}$ Ions in Y Type Hexagonal Ferrite at $293^{\circ}$ K. Journal of Experimental and Theoretical Physics, 60, 1363-1370.

[4] Sizov, R.A. (2008) Electric and Magnetic Spinor Particles as Structure-Forming Components of Mass and Electromagnetic Source Gravitation. Akademizdat-Center "Science", Moscow, 260 p.

[5] Sizov, R.A. (2015) Real Magnetic Poles (Magnetic Charges) in the Physics of Magnetism, Gravitation and Levitation. Journal of Modern Physics, 6, 1013-1022. https://doi.org/10.4236/jmp.2015.68106

[6] Ehrenhaft, F. (1942) Photophoresis and Its Interpretation by Electric and Magnetic Ions. Journal of the Franklin Institute, 233, 235-256. https://doi.org/10.1016/S0016-0032(42)90311-9

[7] Sizov, R.A. (2017) "Electrical" and "Magnetic" Worlds in Universe. Journal of Modern Physics, 8, 1072-1086. https://doi.org/10.4236/jmp.2017.87069

[8] Dirac, P.A.M. (1931) Quantized Singularities in the Electromagnetic Field. Proceedings of the Royal Society A, 133, 60-72. https://doi.org/10.1098/rspa.1931.0130

[9] Sizov, R.A. (2015) Dirac's "Holes" Are the True Antielectrons and Real Particles of Antimatter. Journal of Modern Physics, 6, 2280-2289.

https://doi.org/10.4236/jmp.2015.615232

[10] Sizov, R.A. (2018) Nuclear Physics with Magnetic Charges. Journal of Modern Physics, 9, 145-171. https://doi.org/10.4236/jmp.2018.92010

[11] Sizov, R.A. (2016) Electromagnetic Shells of Atoms and the Periodic System of Elements. Journal of Modern Physics, 7, 2374-2397.

https://doi.org/10.4236/jmp.2016.716206

[12] Maxwell, J.C. (1873) Treatise on Electricity and Magnetism. Volume 1-2, Clarendon Press, Oxford.

[13] Sizov, R.A. (2019) The Electrical Magnetism of Maxwell (1873) Is the "Crooked Mirror" of Physical Science. Applied Physics Research, 11, 49-55. https://doi.org/10.5539/apr.v11n3p49

[14] Sizov, R.A. (2018) Real Magnetic Charges as a Negation of the Electric Magnetism Maxwell and Electrified Dirac's Monopole. Physics \& Astronomy International Journal, 2, 17-20.

[15] Sizov, R.A. (2011/2012) Matter, Antimatter and Energo-Medium Is Physical Triad of the Real World. Akademizdat-Center "Science”, Moscow, $192 \mathrm{p}$.

[16] Sizov, R.A. (2016) World Physical Triad: Matter, Antimatter and "Dark Energy" in the Processes of Climatic Changes on the Earth. Journal of Modern Physics, 7, 558-572. https://doi.org/10.4236/jmp.2016.76059

[17] Sizov, R.A. (2012) Levitation as a Spread Force Manifestation of Natural Ferrogravitation. Akademizdat-Center "Science", Moscow, 96 p.

[18] Sizov, R.A. (2015) Real Magnetic Charges in the Substance, Ferrogravitation and Technical Levitation. Journal of Modern Physics, 6, 1591-1601. https://doi.org/10.4236/jmp.2015.611161

[19] Sizov, R.A. (2019) Intra-Atomic Gravitational Shielding (Lensing), Nuclear Forces and Radioactivity. Journal of Modern Physics, 10, 59-73. https://doi.org/10.4236/jmp.2019.101006 


\section{Appendix}

The author's research has shown that the generally accepted concept of mutual electrostatic "attraction" of ions (as well as their "repulsion") is the result of superficial perception by scientists of the corresponding physical manifestations that are usually observed in practice.

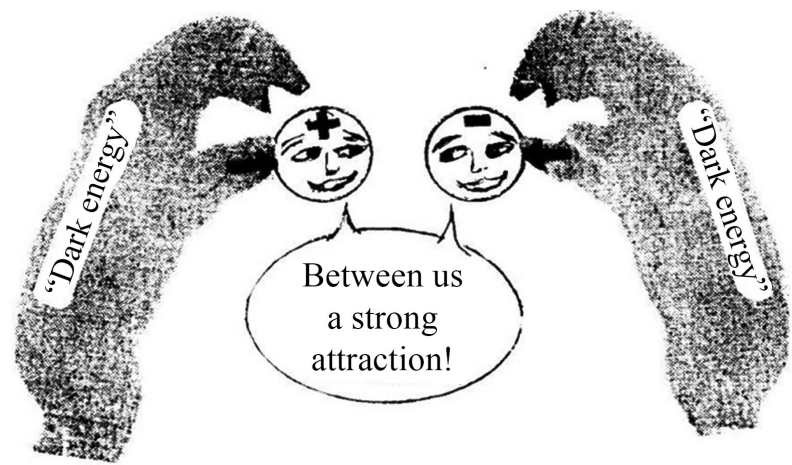

In fact, so-called the attraction between ions charged of charges opposite signs is realized so as shown in the figure, that is, using the power of "bears". 